IJMMS 28:10 (2001) 581-607

PII. S0161171201006706

http://ijmms.hindawi.com

(c) Hindawi Publishing Corp.

\title{
A MATHEMATICAL ANALYSIS OF THERMAL EXPLOSIONS
}

\author{
KAZUAKI TAIRA
}

(Received 16 February 2001)

\begin{abstract}
This paper is devoted to the study of semilinear degenerate elliptic boundary value problems arising in combustion theory which obey the simple Arrhenius rate law and a general Newton law of heat exchange. We prove that ignition and extinction phenomena occur in the stable steady temperature profile at some critical values of a dimensionless rate of heat production.
\end{abstract}

2000 Mathematics Subject Classification. 35J65, 80A25.

1. Introduction and main results. In a reacting material undergoing an exothermic reaction in which reactant consumption is neglected, heat is being produced in accordance with Arrhenius rate law and Newtonian cooling. Thermal explosions occur when the reactions produce heat too rapidly for a stable balance between heat production and heat loss to be preserved. In this paper, we are concerned with the localization of the values of a dimensionless heat evolution rate at which such critical phenomena as ignition and extinction occur. For detailed studies of thermal explosions, the reader might be referred to Aris [3, 4], Bebernes-Eberly [5], Boddington-Gray-Wake [6], and Warnatz-Maas-Dibble [22].

Let $D$ be a bounded domain of Euclidean space $\mathbb{R}^{N}, N \geq 2$, with smooth boundary $\partial D$; its closure $\bar{D}=D \cup \partial D$ is an $N$-dimensional, compact smooth manifold with boundary. We let

$$
A u(x)=-\sum_{i=1}^{N} \frac{\partial}{\partial x_{i}}\left(\sum_{j=1}^{N} a^{i j}(x) \frac{\partial u}{\partial x_{i}}(x)\right)+c(x) u(x)
$$

be a second-order, elliptic differential operator with real coefficients such that:

(1) $a^{i j}(x) \in C^{\infty}(\bar{D})$ with $a^{i j}(x)=a^{j i}(x), 1 \leq i, j \leq N$, and there exists a constant $a_{0}>0$ such that

$$
\sum_{i, j=1}^{N} a^{i j}(x) \xi_{i} \xi_{j} \geq a_{0}|\xi|^{2}, \quad x \in \bar{D}, \xi \in \mathbb{R}^{N}
$$

(2) $c(x) \in C^{\infty}(\bar{D})$ and $c(x)>0$ in $D$.

In this paper, we consider the following semilinear elliptic boundary value problem stimulated by a small fuel loss steady-state model in combustion theory:

$$
A u=\lambda \exp \left[\frac{u}{1+\varepsilon u}\right] \quad \text { in } D, \quad B u=a\left(x^{\prime}\right) \frac{\partial u}{\partial v}+\left(1-a\left(x^{\prime}\right)\right) u=0 \quad \text { on } \partial D
$$




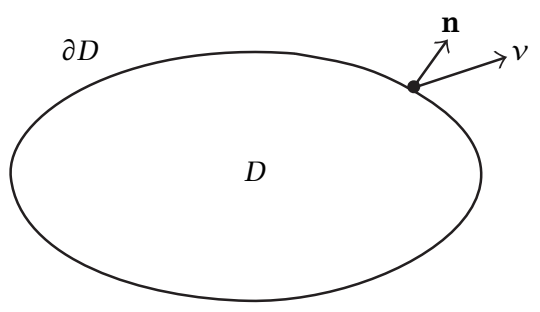

FIGURE 1.1

Here:

(1) $\lambda$ and $\varepsilon$ are positive parameters.

(2) $a\left(x^{\prime}\right) \in C^{\infty}(\partial D)$ and $0 \leq a\left(x^{\prime}\right) \leq 1$ on $\partial D$.

(3) $\partial / \partial v$ is the conormal derivative associated with the operator $A$

$$
\frac{\partial}{\partial v}=\sum_{i, j=1}^{N} a^{i j}\left(x^{\prime}\right) n_{j} \frac{\partial}{\partial x_{i}},
$$

where $\mathbf{n}=\left(n_{1}, n_{2}, \ldots, n_{N}\right)$ is the unit exterior normal to the boundary $\partial D$ (see Figure 1.1).

The nonlinear term

$$
f(t):=\exp \left[\frac{t}{1+\varepsilon t}\right]
$$

describes the temperature dependence of reaction rate for exothermic reactions obeying the simple Arrhenius rate law in circumstances in which heat flow is purely conductive, and the parameter $\varepsilon$ is a dimensionless inverse measure of the Arrhenius activation energy or a dimensionless ambient temperature. The equation

$$
A u=\lambda \exp \left[\frac{u}{1+\varepsilon u}\right]=\lambda f(u) \text { in } D
$$

represents heat balance with reactant consumption ignored, where the function $u$ is a dimensionless temperature excess of a combustible material and the parameter $\lambda$, called the Frank-Kamenetskii parameter, is a dimensionless rate of heat production.

On the other hand, the boundary condition

$$
B u=a\left(x^{\prime}\right) \frac{\partial u}{\partial \mathbf{n}}+\left(1-a\left(x^{\prime}\right)\right) u=0 \quad \text { on } \partial D
$$

represents the exchange of heat at the surface of the reactant by Newtonian cooling. Moreover the boundary condition $\mathrm{Bu}$ is called the isothermal condition (or Dirichlet condition) if $a\left(x^{\prime}\right) \equiv 0$ on $\partial D$, and is called the adiabatic condition (or Neumann condition) if $a\left(x^{\prime}\right) \equiv 1$ on $\partial D$. It should be emphasized that problem (1.3) becomes a degenerate boundary value problem from an analytical point of view. This is due to the fact that the so-called Shapiro-Lopatinskii complementary condition is violated at the points $x^{\prime} \in \partial D$ where $a\left(x^{\prime}\right)=0$. In the non-degenerate case or one-dimensional case, problem (1.3) has been studied by many authors (see Brown-Ibrahim-Shivaji [7], 


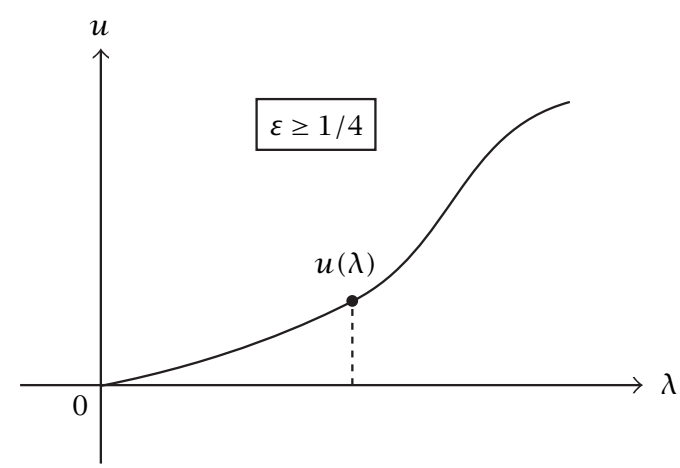

FIGURE 1.2

Cohen [8], Cohen-Laetsch [9], Parter [15], Tam [21], Wiebers [23, 24], and WilliamsLeggett [25]).

A function $u(x) \in C^{2}(\bar{D})$ is called a solution of problem (1.3) if it satisfies the equation $A u-\lambda f(u)=0$ in $D$ and the boundary condition $B u=0$ on $\partial D$. A solution $u(x)$ is said to be positive if it is positive everywhere in $D$.

This paper is devoted to the study of the existence of positive solutions of problem (1.3), and is an expanded and revised version of the previous paper Taira-Umezu [20]. First it follows from an application of Taira-Umezu [19, Theorem 1] that problem (1.3) has at least one positive solution $u(\lambda) \in C^{2}(\bar{D})$ for each $\lambda>0$. Furthermore, by [18, Example 7] we know that problem (1.3) has a unique positive solution $u(\lambda) \in C^{2}(\bar{D})$ for each $\lambda>0$ if $\varepsilon \geq 1 / 4$. In other words, if the activation energy is so low that the parameter $\varepsilon$ exceeds the value $1 / 4$, then only a smooth progression of reaction rate with imposed ambient temperature can occur; such a reaction may be very rapid but it is only accelerating and lacks the discontinuous change associated with criticality and ignition. The situation may be represented schematically by Figure 1.2 (cf. BoddingtonGray-Wake [6, Figure 6]).

The purpose of this paper is to study the case where $0<\varepsilon<1 / 4$. Our main result gives sufficient conditions for problem (1.3) to have three positive solutions, which suggests that the bifurcation curve of problem (1.3) is $S$-shaped (see Figure 1.4).

First, to state our multiplicity theorem for problem (1.3) we introduce a function

$$
v(t):=\frac{t}{f(t)}=\frac{t}{\exp [t /(1+\varepsilon t)]}, \quad t \geq 0 .
$$

It is easy to see (see Figure 1.3) that if $0<\varepsilon<1 / 4$, then the function $v(t)$ has a unique local maximum at $t=t_{1}(\varepsilon)$

$$
t_{1}(\varepsilon)=\frac{1-2 \varepsilon-\sqrt{1-4 \varepsilon}}{2 \varepsilon^{2}},
$$

and has a unique local minimum at $t=t_{2}(\varepsilon)$

$$
t_{2}(\varepsilon)=\frac{1-2 \varepsilon+\sqrt{1-4 \varepsilon}}{2 \varepsilon^{2}} .
$$




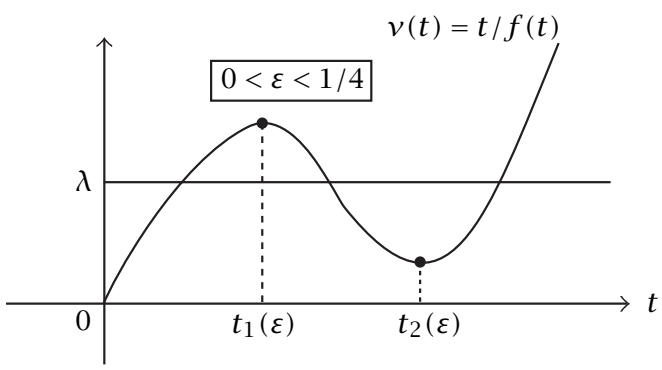

FIGURE 1.3

Wiebers $[23,24]$ proved a rigorous qualitative connection between the positive solution set of problem (1.3) and the solution set of the so-called Semenov approximation $\lambda=v(t), \lambda>0$

On the other hand, let $\phi(x) \in C^{\infty}(\bar{D})$ be the unique positive solution of the linear boundary value problem

$$
A \phi=1 \quad \text { in } D, \quad B \phi=0 \quad \text { on } \partial D \text {, }
$$

and let

$$
\|\phi\|_{\infty}=\max _{\bar{D}} \phi(x) .
$$

Now we can state our multiplicity theorem for problem (1.3).

THEOREM 1.1. There exists a constant $\beta>0$, independent of $\varepsilon$, such that if $0<\varepsilon<1 / 4$ is so small that

$$
\frac{v\left(t_{2}(\varepsilon)\right)}{\beta}<\frac{v\left(t_{1}(\varepsilon)\right)}{\|\phi\|_{\infty}}
$$

then problem (1.3) has at least three distinct positive solutions $u_{1}(\lambda), u_{2}(\lambda), u_{3}(\lambda)$ for all $\lambda$ satisfying the condition

$$
\frac{v\left(t_{2}(\varepsilon)\right)}{\beta}<\lambda<\frac{v\left(t_{1}(\varepsilon)\right)}{\|\phi\|_{\infty}} .
$$

It should be noticed that, as $\varepsilon \downarrow 0$, the local maximum $v\left(t_{1}(\varepsilon)\right)$ and the local minimum $v\left(t_{2}(\varepsilon)\right)$ behave, respectively, as follows:

$$
v\left(t_{1}(\varepsilon)\right) \sim \exp \left[\frac{-1}{1+\varepsilon}\right], \quad v\left(t_{2}(\varepsilon)\right) \sim \frac{1}{\varepsilon^{2}} \exp \left[\frac{-1}{\varepsilon+\varepsilon^{2}}\right] .
$$

This implies that condition (1.13) makes sense.

Theorem 1.1 is a generalization of Wiebers [23, Theorem 4.3] and [24, Theorem 3.1] to the degenerate case. The situation may be represented schematically by Figure 1.4 (cf. Boddington-Gray-Wake [6, Figure 6]).

Secondly, we state two existence and uniqueness theorems for problem (1.3). Let $\lambda_{1}$ be the first eigenvalue of the linear eigenvalue problem

$$
A u=\lambda u \quad \text { in } D, \quad B u=0 \quad \text { on } \partial D .
$$




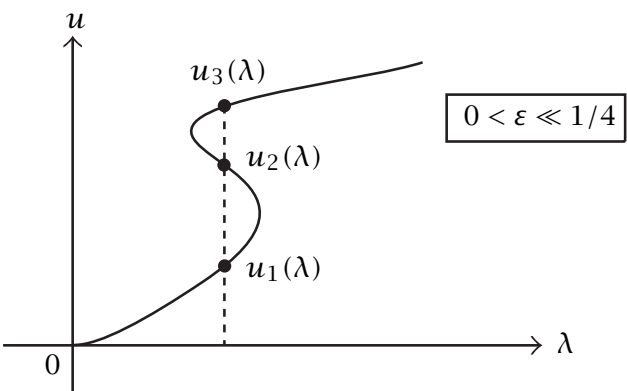

FIGURE 1.4

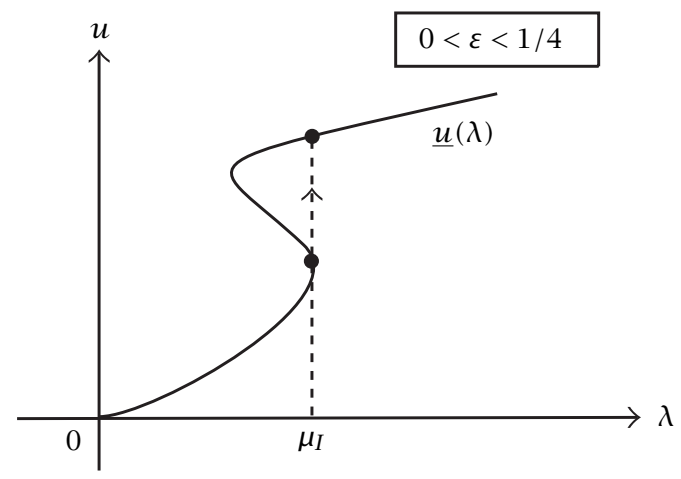

FIGURE 1.5

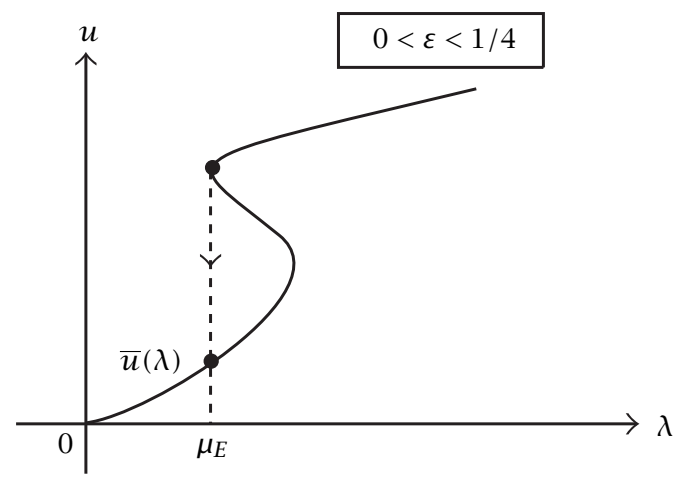

FIGURE 1.6

The next two theorems assert that problem (1.3) is uniquely solvable for $\lambda$ sufficiently small and sufficiently large if $0<\varepsilon<1 / 4$ (see Figures 1.5 and 1.6). 
THEOREM 1.2. Let $0<\varepsilon<1 / 4$. If the parameter $\lambda$ is so small that

$$
0<\lambda<\frac{\lambda_{1} \exp [(2 \varepsilon-1) / \varepsilon]}{4 \varepsilon^{2}},
$$

then problem (1.3) has a unique positive solution $u(\lambda) \in C^{2}(\bar{D})$.

THEOREM 1.3. Let $0<\varepsilon<1 / 4$. There exists a constant $\Lambda>0$, independent of $\varepsilon$, such that if the parameter $\lambda$ is so large that $\lambda>\Lambda$, then problem (1.3) has a unique positive solution $u(\lambda) \in C^{2}(\bar{D})$.

Theorems 1.2 and 1.3 are generalizations of Wiebers [23, Theorems 2.6 and 2.9] to the degenerate case, respectively, although we only treat the nonlinear term $f(t)=$ $\exp [t /(1+\varepsilon t)]$.

Moreover, if $\phi(x)$ is the unique positive solution of problem (1.11), then we can prove the following asymptotic behavior of positive solutions of problem (1.3) as $\lambda \downarrow 0$ and as $\lambda \uparrow \infty$, for any $0<\varepsilon<1 / 4$.

THEOREM 1.4. Let $0<\varepsilon<1 / 4$, and let $u(\lambda) \in C^{2}(\bar{D})$ be the unique positive solution of problem (1.3) for $\lambda$ sufficiently small or for $\lambda$ sufficiently large as in Theorems 1.2 and 1.3. Then the following asymptotics hold:

$$
u(\lambda) \sim \lambda \phi(x) \text { as } \lambda \downarrow 0, \quad u(\lambda) \sim \lambda e^{1 / \varepsilon} \phi(x) \quad \text { as } \lambda \uparrow \infty .
$$

More precisely,

$$
\begin{aligned}
& \frac{u(\lambda)}{\lambda} \longrightarrow \phi(x) \quad \text { in } C^{1}(\bar{D}) \text { as } \lambda \downarrow 0, \\
& \frac{u(\lambda)}{\lambda} \longrightarrow e^{1 / \varepsilon} \phi(x) \quad \text { in } C^{1}(\bar{D}) \text { as } \lambda \uparrow \infty .
\end{aligned}
$$

By virtue of Theorems 1.1, 1.2, and 1.3, we can define two positive numbers $\mu_{I}$ and $\mu_{E}$ by the formulas

$$
\begin{aligned}
& \mu_{I}=\inf \{\mu>0: \text { problem (1.3) is uniquely solvable for each } \lambda>\mu\}, \\
& \mu_{E}=\sup \{\mu>0 \text { : problem (1.3) is uniquely solvable for each } 0<\lambda<\mu\} .
\end{aligned}
$$

Then certain physical conclusions may be drawn (cf. [5, 22]). If the system is in a state corresponding to a point on the lower branch and if $\lambda$ is slowly increased, then the solution can be expected to change smoothly until the point $\mu_{I}$ is reached. Rapid transition to the upper branch will then presumably occur, corresponding to ignition. A subsequent slow decrease in $\lambda$ is likewise anticipated to produce a smooth decrease in burning rate until extinction occurs at the point $\mu_{E}$. In other words, the minimal positive solution $\underline{u}(\lambda)$ is continuous for $\lambda>\mu_{I}$ but is not continuous at $\lambda=\mu_{I}$, while the maximal positive solution $\bar{u}(\lambda)$ is continuous for $0<\lambda<\mu_{E}$ but is not continuous at $\lambda=\mu_{E}$. The situation may be represented schematically by Figures 1.5 and 1.6 (cf. Boddington-Gray-Wake [6, Figure 6]).

By the maximum principle and the boundary point lemma, we can obtain from the variational formula (4.5) that the first eigenvalue $\lambda_{1}=\lambda_{1}(a)$ of problem (1.16) satisfies the inequalities

$$
\lambda_{1}(1)<\lambda_{1}(a)<\lambda_{1}(0)
$$


Moreover, it follows that the unique solution $\phi=\phi_{(a)}$ of problem (1.11) satisfies the inequalities

$$
\phi_{(0)}<\phi_{(a)}<\phi_{(1)} \text { in } D
$$

so that,

$$
\frac{1}{\left\|\phi_{(1)}\right\|_{\infty}}<\frac{1}{\left\|\phi_{(a)}\right\|_{\infty}}<\frac{1}{\left\|\phi_{(0)}\right\|_{\infty}} .
$$

On the other hand, we find from formula (3.29) that the critical value $\beta=\beta(a)$ in Theorem 1.1 satisfies the inequalities

$$
\frac{1}{\beta(1)} \leq \frac{1}{\beta(a)} \leq \frac{1}{\beta(0)},
$$

and further from formulas (5.54) and (5.63) that the critical value $\Lambda=\Lambda(a)$ in Theorem 1.3 depends essentially on the first eigenvalue $\lambda_{1}=\lambda_{1}(a)$.

Therefore, we can conclude that the extinction phenomenon in the isothermal condition case occurs at the largest critical value $\mu_{E}(0)$, while the extinction phenomenon in the adiabatic condition case occurs at the smallest critical value $\mu_{E}(1)$. Similarly, we find that ignition phenomenon in the adiabatic condition case occurs at the smallest critical value $\mu_{I}(1)$, while the ignition phenomenon in the isothermal condition case occurs at the largest critical value $\mu_{I}(0)$.

REMARK 1.5. Minamoto-Yamamoto-Nakao [14] studied the case where $D$ is the unit ball in $\mathbb{R}^{3}$ under the Dirichlet condition $a\left(x^{\prime}\right) \equiv 0$ on $\partial D$. The following numerical analysis of the critical value $\mu_{I}(0)$ is due to them (cf. [15, 24]):

\begin{tabular}{ll}
\hline$\varepsilon$ & $\mu_{I}(0)$ \\
\hline 0.01 & 3.359 \\
0.02 & 3.399 \\
0.05 & 3.526 \\
0.20 & 4.510 \\
\hline
\end{tabular}

The rest of this paper is organized as follows. In Section 2 we collect the basic definitions and notions about the theory of positive mappings in ordered Banach spaces. This section is adapted from Amann [2]. Section 3 is devoted to the proof of Theorem 1.1. We reduce the study of problem (1.3) to the study of a nonlinear operator equation in an appropriate order Banach space just as in Taira-Umezu [20]. The methods developed here are based on a multiple positive fixed point technique formulated by Leggett-Williams [13] (see Lemma 3.2). This technique is intended to reduce the usually difficult task of establishing the existence of multiple positive solutions of problem (1.3) to the verification of a few elementary conditions on the nonlinear term $f(u)$ and the resolvent $K$, just as in Wiebers [23, Theorem 4.3]. In Section 4 we make use of a variational formula (4.5) to prove Theorem 1.2, since the linear operator $\mathfrak{U}$ associated with the eigenvalue problem (1.16) is selfadjoint in the Hilbert space $L^{2}(D)$. In Section 5 the proof of Theorem 1.3 may be carried out by adapting the proof of Wiebers [23, Theorems 2.6 and 2.9] to the degenerate case. In particular, we establish 
an a priori estimate for all positive solutions of problem (1.3) (see Proposition 5.1) which plays an important role in the proof of Theorem 1.3. The final Section 6 is devoted to the proof of Theorem 1.4. Our proof of Theorem 1.4 is inspired by Dancer [10, Theorem 1].

2. Ordered Banach spaces and the fixed point index. One of the most important tools in nonlinear functional analysis is the Leray-Schauder degree of a compact perturbation of the identity mapping of a Banach spaces into itself. In connection with nonlinear mappings in ordered Banach spaces, it is natural to consider mappings defined on open subsets of the positive cone. Since the positive cone is a retract of the Banach space, one can define a fixed point index for compact mappings on the positive cone as is shown in Amann [2, Section 11].

2.1. Ordered Banach spaces. Let $X$ be a nonempty set. An ordering $\leq$ in $X$ is a relation in $X$ that is reflexive, transitive and antisymmetric. A nonempty set together with an ordering is called an ordered set.

Let $V$ be a real vector space. An ordering $\leq$ in $V$ is said to be linear if the following two conditions are satisfied:

(i) If $x, y \in V$ and $x \leq y$, then we have $x+z \leq y+z$ for all $z \in V$.

(ii) If $x, y \in V$ and $x \leq y$, then we have $\alpha x \leq \alpha y$ for all $\alpha \geq 0$.

A real vector space together with a linear ordering is called an ordered vector space. If $x, y \in V$ and $x \leq y$, then the set $[x, y]=\{z \in X: x \leq z \leq y\}$ is called an order interval.

If we let

$$
Q=\{x \in V: x \geq 0\},
$$

then it is easy to verify that the set $Q$ has the following two conditions:

(iii) If $x, y \in Q$, then $\alpha x+\beta y \in Q$ for all $\alpha, \beta \geq 0$.

(iv) If $x \neq 0$, then at least one of $x$ and $-x$ does not belong to $Q$.

The set $Q$ is called the positive cone of the ordering $\leq$.

Let $E$ be a Banach space with a linear ordering $\leq$. The Banach space $E$ is called an ordered Banach space if the positive cone $Q$ is closed in $E$. It is to be expected that the topology and the ordering of an ordered Banach space are closely related if the norm is monotone: if $0 \leq u \leq v$, then $\|u\| \leq\|v\|$.

2.2. Retracts and retractions. Let $X$ be a metric space. A nonempty subset $A$ of $X$ is called a retract of $X$ if there exists a continuous map $r: X \rightarrow A$ such that the restriction $\left.r\right|_{A}$ to $A$ is the identity map. The map $r$ is called a retraction.

The next theorem, due to Dugundji $[11,12]$, gives a sufficient condition in order that a subset of a Banach space be a retract.

THEOREM 2.1. Every nonempty closed convex subset of a Banach space $E$ is a retract of $E$.

2.3. The fixed point index. Let $E$ and $F$ be Banach spaces, and let $A$ be a nonempty subset of $E$. A map $f: A \rightarrow F$ is said to be compact if it is continuous and the image $f(A)$ is relatively compact in $F$. 
Theorem 2.1 tells us that the positive cone $Q$ is a retract of the Banach space $E$. Therefore one can define a fixed point index for compact mappings defined on the positive cone; more precisely, the next theorem asserts that one can define a fixed point index for compact maps on closed subsets of a retract of $E$.

THEOREM 2.2. Let $E$ be a Banach space and let $X$ be a retract of $E$. If $U$ is an open subset of $X$ and if $f: \bar{U} \rightarrow X$ is a compact map such that $f(x) \neq x$ for all $x \in \partial U$, then define an integer $i(f, U, X)$ satisfying the following four conditions:

(i) (Normalization): for every constant map $f: \bar{U} \rightarrow U$, we have

$$
i(f, U, X)=1 \text {. }
$$

(ii) (Additivity): for every pair $\left(U_{1}, U_{2}\right)$ of disjoint open subsets of $U$ such that $f(x) \neq$ $x$ for all $x \in \bar{U} \backslash\left(U_{1} \cup U_{2}\right)$, we have

$$
i(f, U, X)=i\left(\left.f\right|_{\bar{U}_{1}}, U_{1}, X\right)+i\left(\left.f\right|_{\bar{U}_{2}}, U_{2}, X\right) .
$$

(iii) (Homotopy invariance): for every bounded, closed interval $\Lambda$ and every compact map $h: \Lambda \times \bar{U} \rightarrow X$ such that $h(\lambda, x) \neq x$ for all $(\lambda, x) \in \Lambda \times \partial U$, the integer

$$
i(h(\lambda, \cdot), U, X)
$$

is well defined and independent of $\lambda \in \Lambda$.

(iv) (Permanence): if $Y$ is a retract of $X$ and $f(\bar{U}) \subset Y$, then we have

$$
i(f, U, X)=i\left(\left.f\right|_{\overline{U \cap Y}}, U \cap Y, Y\right) .
$$

The integer $i(f, U, X)$ is called the fixed point index of $f$ over $U$ with respect to $X$. In fact, the integer $i(f, U, X)$ is defined by the formula

$$
i(f, U, X)=\operatorname{deg}\left(I-f \circ r, r^{-1}(U), 0\right),
$$

where $r: E \rightarrow X$ is an arbitrary retraction and $\operatorname{deg}\left(I-f \circ r, r^{-1}(U), 0\right)$ is the LeraySchauder degree with respect to zero of the map $I-f \circ r$ defined on the closure of the open subset $r^{-1}(U)$ (see Figure 2.1).

The fixed point index enjoys further important and useful properties.

COROLLARY 2.3. Let $E$ be a Banach space and let $X$ be a retract of $E$. If $U$ is an open subset of $X$ and if $f: \bar{U} \rightarrow X$ is a compact map such that $f(x) \neq x$ for all $x \in \partial U$, then the fixed point index $i(f, U, X)$ has the following two properties:

(v) (Excision): for every open subset $V \subset U$ such that $f(x) \neq x$ for all $x \in \bar{U} \backslash V$, we have

$$
i(f, U, X)=i\left(\left.f\right|_{\bar{V}}, V, X\right) .
$$

(vi) (Solution property): if $i(f, U, X) \neq 0$, then the map $f$ has at least one fixed point in $U$. 


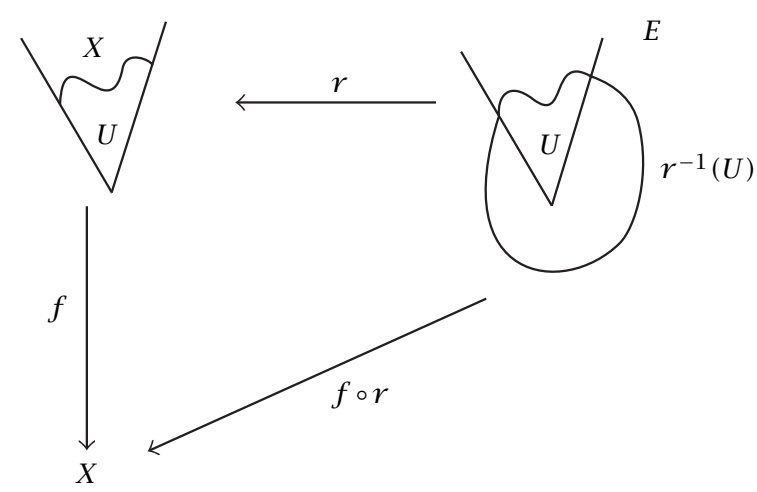

FIGURE 2.1

3. Proof of Theorem 1.1. This section is devoted to the proof of Theorem 1.1. First we transpose the nonlinear problem (1.3) into an equivalent fixed point equation for the resolvent $K$ in an appropriate ordered Banach space, just as in Taira-Umez [20].

To do this, we consider the following linearized problem: for any given function $g \in L^{p}(D)$, find a function $u$ in $D$ such that

$$
A u=g \quad \text { in } D, \quad B u=0 \quad \text { on } \partial D .
$$

Then we have the following existence and uniqueness theorem for problem (3.1) in the framework of $L^{p}$ spaces (see [17, Theorem 1]).

THEOREM 3.1. Let $1<p<\infty$. Then the mapping

$$
\mathscr{A}: W_{B}^{2, p}(D) \longrightarrow L^{p}(D), \quad u \longmapsto A u
$$

is an algebraic and topological isomorphism. Here $W^{2, p}(D)$ is the usual Sobolev space of $L^{p}$ style and

$$
W_{B}^{2, p}(D)=\left\{u \in W^{2, p}(D): B u=0 \text { on } \partial D\right\} .
$$

(I) By Theorem 3.1, we can introduce a continuous linear operator

$$
K: L^{p}(D) \longrightarrow W_{B}^{2, p}(D)
$$

as follows: for any $g \in L^{p}(D)$, the function $u=K g \in W^{2, p}(D)$ is the unique solution of problem (3.1). Then, by the Ascoli-Arzelà theorem we find that the operator $K$, considered as

$$
K: C(\bar{D}) \longrightarrow C^{1}(\bar{D})
$$

is compact. Indeed it follows from an application of Sobolev's imbedding theorem that $W^{2, p}(D)$ is continuously imbedded into $C^{2-N / p}(\bar{D})$ for all $N<p<\infty$.

For $u, v \in C(\bar{D})$, we write $u \geq v$ if $u(x) \geq v(x)$ in $\bar{D}$. Then the space $C(\bar{D})$ is an ordered Banach space with the linear ordering $\succeq$, and with the positive cone

$$
P=\{u \in C(\bar{D}): u \succeq 0\}
$$


For $u, v \in C(\bar{D})$, the notation $u \succ v$ means that $u-v \in P \backslash\{0\}$. Then it follows from an application of the maximum principle (cf. [16]) that the resolvent $K$ is strictly positive, that is, $K g$ is positive everywhere in $D$ if $g \succ 0$ (see [18, Lemma 2.7]). Moreover it is easy to verify that a function $u(x)$ is a solution of problem (1.3) if and only if it satisfies the nonlinear operator equation

$$
u=\lambda K(f(u)) \quad \text { in } C(\bar{D}) .
$$

(II) The proof of Theorem 1.1 is based on the following result on multiple positive fixed points of nonlinear operators on ordered Banach spaces essentially due to Leggett-Williams [13] (cf. Wiebers [23, Lemma 4.4]).

LEMMA 3.2. Let $(X, Q, \preceq)$ be an ordered Banach space such that the positive cone $Q$ has nonempty interior. Moreover, let $\eta: Q \rightarrow[0, \infty)$ be a continuous and concave functional and let $G$ be a compact mapping of $Q_{\tau}:=\{w \in Q:\|w\| \leq \tau\}$ into $Q$ for some constant $\tau>0$ such that

$$
\|G(w)\|<\tau \quad \forall w \in Q_{\tau} \text { satisfying }\|w\|=\tau .
$$

Assume that there exist constants $0<\delta<\tau$ and $\sigma>0$ such that the set

$$
W:=\left\{w \in \stackrel{\circ}{Q}_{\tau}: \eta(w)>\sigma\right\}
$$

is nonempty, where $\AA$ denotes the interior of a subset $A$ of $Q$, and that

$$
\begin{gathered}
\|G(w)\|<\delta \quad \forall w \in Q_{\delta} \text { satisfying }\|w\|=\delta, \\
\eta(w)<\sigma \quad \forall w \in Q_{\delta}, \\
\eta(G(w))>\sigma \quad \forall w \in Q_{\tau} \text { satisfying } \eta(w)=\sigma .
\end{gathered}
$$

Then the mapping $G$ has at least three distinct fixed points.

Proof. Let $i(G, U, Q)$ denote the fixed point index of the mapping $G(\cdot)$ over an open subset $U$ with respect to the positive cone $Q$ as is stated in Theorem 2.2.

We let

$$
\tilde{G}(w)=t G(w)+(1-t) \cdot 0=t G(w), \quad 0 \leq t \leq 1 .
$$

Then we have, by condition (3.8),

$$
\|\tilde{G}(w)\|=t\|G(w)\|<\tau \quad \forall\|w\|=\tau .
$$

This implies that

$$
w \neq \tilde{G}(w) \quad \forall w \in \partial \stackrel{\circ}{Q}_{\tau} .
$$

Therefore, by the homotopy invariance (iii) and the normalization (i) of the index we obtain that

$$
i\left(G, \stackrel{\circ}{Q}_{T}, Q\right)=i\left(0, \stackrel{\circ}{Q}_{\tau}, Q\right)=1
$$


Similarly, by condition (3.10) it follows that

$$
i\left(G, \stackrel{\circ}{Q}_{\delta}, Q\right)=1 .
$$

Next we show that

$$
i(G, W, Q)=1 .
$$

By the continuity of $\eta$ we find that the set $W$ is open, so that the index $i(G, W, Q)$ is well defined. Moreover, by condition (3.9) one can choose a point $w_{0} \in W$. We notice that if $w \in \partial W$, then it follows that either $\|w\|=\tau$ or $\eta(w)=\sigma$.

(i) First, if $\|w\|=\tau$, we let

$$
\hat{G}(w)=t G(w)+(1-t) w_{0}, \quad 0 \leq t \leq 1 .
$$

Then we have, by condition (3.8),

$$
\|\hat{G}(w)\| \leq t\|G(w)\|+(1-t)\left\|w_{0}\right\|<\tau .
$$

This implies that

$$
w \neq \hat{G}(w) \quad \forall\|w\|=\tau .
$$

(ii) Secondly, if $\eta(w)=\sigma$, it follows from condition (3.12) that

$$
\begin{aligned}
\eta(\hat{G}(w)) & =\eta\left(t G(w)+(1-t) w_{0}\right) \\
& \geq t \eta(G(w))+(1-t) \eta\left(w_{0}\right) \\
& >t \sigma+(1-t) \sigma=\sigma
\end{aligned}
$$

since the functional $\eta$ is concave. Hence we have

$$
w \neq \hat{G}(w) \quad \forall \eta(w)=\sigma .
$$

Summing up, we have proved that

$$
w \neq \hat{G}(w) \quad \forall w \in \partial W
$$

Therefore, by the homotopy invariance (iii) and the normalization (i) of the index it follows that

$$
i(G, W, Q)=i\left(w_{0}, W, Q\right)=1
$$

Now, if we let

$$
U=\left\{w \in \stackrel{\circ}{Q}_{\tau}: \eta(w)<\sigma,\|w\|>\delta\right\}
$$

then we find from condition (3.11) that the sets $\stackrel{\circ}{Q}_{\delta}, U$, and $W$ are disjoint (see Figure 3.1).

Thus, by the additivity (ii) of the index it follows from assertions (3.16), (3.17), and (3.18) that

$$
i(G, U, Q)=i\left(G, \stackrel{\circ}{Q}_{\tau}, Q\right)-i\left(G, \stackrel{\circ}{Q}_{\delta}, Q\right)-i(G, W, Q)=-1
$$




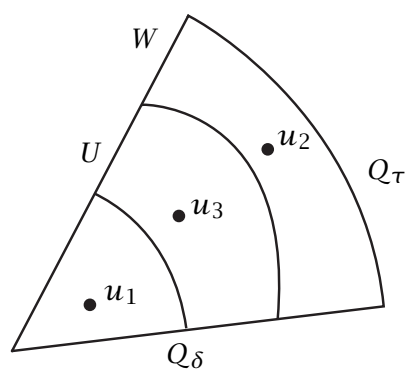

FIGURE 3.1

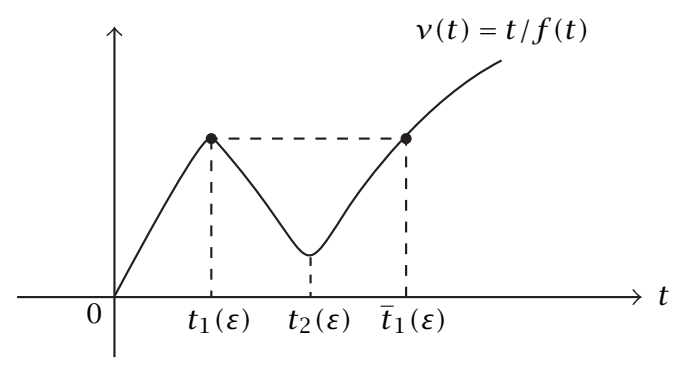

FIGURE 3.2

Therefore, by the solution property (vi) of the index we can find three distinct fixed points $u_{1}, u_{2}, u_{3}$ of $G(\cdot)$ such that

$$
u_{1} \in \stackrel{\circ}{Q}_{\delta}, \quad u_{2} \in W, \quad u_{3} \in U .
$$

The proof of Lemma 3.2 is now complete.

(III) End of proof of Theorem 1.1. The proof of Theorem 1.1 may be carried out just as in the proof of Wiebers [23, Theorem 4.3].

Let $\mathscr{B}$ be the set of all subdomains $\Omega$ of $D$ with smooth boundary such that $\operatorname{dist}(\Omega, \partial D)>0$, and let

$$
\beta=\sup _{\Omega \in \mathscr{P}} C_{\Omega}, \quad C_{\Omega}=\inf _{x \in \Omega}\left(K \chi_{\Omega}\right)(x),
$$

where $\chi_{\Omega}$ denotes the characteristic function of a set $\Omega$. It is easy to see that the constant $\beta$ is positive, since the resolvent $K$ of problem (3.1) is strictly positive.

Since $\lim _{t \rightarrow \infty} v(t)=\lim _{t \rightarrow \infty} t / f(t)=\infty$, one can find a constant $\bar{t}_{1}(\varepsilon)$ such that (see Figure 3.2)

$$
\bar{t}_{1}(\varepsilon)=\min \left\{t>t_{2}(\varepsilon): v(t)=v\left(t_{1}(\varepsilon)\right)\right\} .
$$

It should be noticed that

$$
t_{1}(\varepsilon)<t_{2}(\varepsilon)<\bar{t}_{1}(\varepsilon),
$$


and that

$$
v\left(t_{1}(\varepsilon)\right)=v\left(\bar{t}_{1}(\varepsilon)\right)=\frac{\bar{t}_{1}(\varepsilon)}{f\left(\bar{t}_{1}(\varepsilon)\right)} .
$$

Now we apply Lemma 3.2 with

$$
\begin{gathered}
X:=C(\bar{D}), \quad Q:=P=\{u \in C(\bar{D}): u \geq 0\}, \\
G(\cdot):=\lambda K(f(\cdot)), \quad \delta:=t_{1}(\varepsilon), \quad \sigma:=t_{2}(\varepsilon), \quad \tau:=\bar{t}_{1}(\varepsilon) .
\end{gathered}
$$

To do this, it suffices to verify that conditions of Lemma 3.2 are fulfilled for all $\lambda$ satisfying condition (1.14).

(III-a) If $t>0$, we let

$$
P(t)=\left\{u \in P:\|u\|_{\infty} \leq t\right\} .
$$

If $u \in P\left(\bar{t}_{1}(\varepsilon)\right)$ and $\|u\|_{\infty}=\bar{t}_{1}(\varepsilon)$ and if $\phi(x)=K 1(x)$ is the unique solution of problem (1.11), then it follows from condition (1.14) and formula (3.32) that

$$
\begin{aligned}
\|\lambda K(f(u))\|_{\infty} & <\frac{v\left(t_{1}(\varepsilon)\right)}{\|\phi\|_{\infty}}\|K(f(u))\|_{\infty} \\
& \leq \frac{v\left(t_{1}(\varepsilon)\right)}{\|\phi\|_{\infty}} f\left(\bar{t}_{1}(\varepsilon)\right)\|K 1\|_{\infty} \\
& =v\left(t_{1}(\varepsilon)\right) f\left(\bar{t}_{1}(\varepsilon)\right)=\bar{t}_{1}(\varepsilon),
\end{aligned}
$$

since $f(t)$ is increasing for all $t \geq 0$. This proves that the mapping $\lambda K(f(\cdot))$ satisfies condition (3.8) with $Q_{\tau}:=P\left(\bar{t}_{1}(\varepsilon)\right)$.

Similarly, one can verify that if $u \in P\left(t_{1}(\varepsilon)\right)$ and $\|u\|_{\infty}=t_{1}(\varepsilon)$, then we have

$$
\|\lambda K(f(u))\|_{\infty}<t_{1}(\varepsilon)
$$

This proves that the mapping $\lambda K(f(\cdot))$ satisfies condition (3.10) with $Q_{\delta}:=P\left(t_{1}(\varepsilon)\right.$ ).

(III-b) If $\Omega \in \mathscr{B}$, we let

$$
\eta(u)=\inf _{x \in \Omega} u(x)
$$

Then it is easy to see that $\eta$ is a continuous and concave functional of $P$. If $u \in P\left(t_{1}(\varepsilon)\right)$, then we have

$$
\eta(u) \leq\|u\|_{\infty} \leq t_{1}(\varepsilon)<t_{2}(\varepsilon) .
$$

This verifies condition (3.11) for the functional $\eta$.

(III-c) If we let

$$
W=\left\{u \in \stackrel{\circ}{P}\left(\bar{t}_{1}(\varepsilon)\right): \eta(u)>t_{2}(\varepsilon)\right\},
$$

then we find that

$$
W \supset\left\{u \in P: \frac{\bar{t}_{1}(\varepsilon)}{2} \leq u<\bar{t}_{1}(\varepsilon) \text { on } \bar{D}, \eta(u)>t_{2}(\varepsilon)\right\} \neq \varnothing
$$

since $t_{2}(\varepsilon)<\bar{t}_{1}(\varepsilon)$. This verifies condition (3.9) for the functional $\eta$. 
(III-d) Now, since $\lambda>v\left(t_{2}(\varepsilon)\right) / \beta$, by formula (3.29) one can find a subdomain $\Omega \in \mathscr{B}$ such that

$$
\lambda>\frac{v\left(t_{2}(\varepsilon)\right)}{C_{\Omega}}
$$

If $u \in P\left(\bar{t}_{1}(\varepsilon)\right)$ and $\eta(u)=t_{2}(\varepsilon)$, then we have

$$
\begin{aligned}
\eta(\lambda K(f(u))) & =\inf _{x \in \Omega} \lambda K(f(u))(x) \\
& \geq \inf _{x \in \Omega} \lambda K\left(f(u) \chi_{\Omega}\right)(x) \\
& >\frac{v\left(t_{2}(\varepsilon)\right)}{C_{\Omega}} \inf _{x \in \Omega} K\left(f(u) \chi_{\Omega}\right)(x) .
\end{aligned}
$$

However, since $\inf _{\Omega} u=\eta(u)=t_{2}(\varepsilon)$ and $f(t)$ is increasing for all $t \geq 0$, it follows that

$$
\begin{aligned}
\frac{v\left(t_{2}(\varepsilon)\right)}{C_{\Omega}} \inf _{x \in \Omega} K\left(f(u) \chi_{\Omega}\right)(x) & \geq \frac{v\left(t_{2}(\varepsilon)\right)}{C_{\Omega}} \inf _{x \in \Omega} K\left(f\left(t_{2}(\varepsilon)\right) \chi_{\Omega}\right)(x) \\
& =\frac{v\left(t_{2}(\varepsilon)\right)}{C_{\Omega}} f\left(t_{2}(\varepsilon)\right) \inf _{x \in \Omega}\left(K \chi_{\Omega}\right)(x) \\
& =v\left(t_{2}(\varepsilon)\right) f\left(t_{2}(\varepsilon)\right) \\
& =t_{2}(\varepsilon) .
\end{aligned}
$$

Therefore, combining inequalities (3.42) and (3.43) we obtain that

$$
\eta(\lambda K(f(u)))>t_{2}(\varepsilon) .
$$

This verifies condition (3.12) for the mapping $\lambda K(f(\cdot))$.

The proof of Theorem 1.1 is now complete.

4. Proof of Theorem 1.2. We let

$$
f(t)=\exp \left[\frac{t}{1+\varepsilon t}\right], \quad t \geq 0
$$

If $u_{1}=u_{1}(\lambda)$ and $u_{2}=u_{2}(\lambda)$ are two positive solutions of problem (1.3), then we have, by the mean value theorem,

$$
\begin{aligned}
\int_{D} A\left(u_{1}-u_{2}\right) \cdot\left(u_{1}-u_{2}\right) d x & =\int_{D} \lambda\left(f\left(u_{1}\right)-f\left(u_{2}\right)\right)\left(u_{1}-u_{2}\right) d x \\
& =\lambda \int_{D} G(x)\left(u_{1}-u_{2}\right)^{2} d x
\end{aligned}
$$

where

$$
G(x)=\int_{0}^{1} f^{\prime}\left(u_{2}(x)+\theta\left(u_{1}(x)-u_{2}(x)\right)\right) d \theta .
$$


We will prove Theorem 1.2 by using a variant of variational method. To do this, we introduce an unbounded linear operator $\mathfrak{U}$ from the Hilbert space $L^{2}(D)$ into itself as follows:

(a) The domain of definition $D(\mathfrak{U})$ of $\mathfrak{U}$ is the space

$$
D(\mathfrak{U})=\left\{u \in W^{2,2}(D): B u=0\right\} .
$$

(b) $\mathfrak{L} u=A u, u \in D(\mathfrak{U})$.

Then it follows from [18, Theorem 2.6] that the operator $\mathfrak{U}$ is a positive and selfadjoint operator in $L^{2}(D)$, and has a compact resolvent. Hence we obtain that the first eigenvalue $\lambda_{1}$ of $\mathfrak{U}$ is characterized by the following variational formula:

$$
\lambda_{1}=\min \left\{\int_{D} A u(x) \cdot \overline{u(x)} d x: u \in W^{2,2}(D), \int_{D}|u(x)|^{2} d x=1, B u=0\right\} .
$$

Thus it follows from formulas (4.2) and (4.5) that

$$
\begin{aligned}
\lambda_{1} \int_{D}\left(u_{1}-u_{2}\right)^{2} d x & \leq \int_{D} A\left(u_{1}-u_{2}\right) \cdot\left(u_{1}-u_{2}\right) d x \\
& =\lambda \int_{D} G(x)\left(u_{1}-u_{2}\right)^{2} d x \\
& \leq \lambda \sup f^{\prime}(t) \int_{D}\left(u_{1}-u_{2}\right)^{2} d x
\end{aligned}
$$

However, it is so easy to see that

$$
\sup f^{\prime}(t)=f^{\prime}\left(\frac{1-2 \varepsilon}{2 \varepsilon^{2}}\right)=4 \varepsilon^{2} \exp \left[\frac{1-2 \varepsilon}{\varepsilon}\right] .
$$

Hence, combining formula (4.7) with inequality (4.6) we obtain that

$$
\lambda_{1} \int_{D}\left(u_{1}-u_{2}\right)^{2} d x \leq 4 \lambda \varepsilon^{2} \exp \left[\frac{1-2 \varepsilon}{\varepsilon}\right] \int_{D}\left(u_{1}-u_{2}\right)^{2} d x
$$

Therefore we find that $u_{1}(x) \equiv u_{2}(x)$ in $D$ if the parameter $\lambda$ is so small that condition (1.17) is satisfied, that is, if we have

$$
\lambda_{1}-4 \lambda \varepsilon^{2} \exp \left[\frac{1-2 \varepsilon}{\varepsilon}\right]>0 .
$$

The proof of Theorem 1.2 is complete.

5. Proof of Theorem 1.3. This section is devoted to the proof of Theorem 1.3. Our proof of Theorem 1.3 is based on a method inspired by Wiebers [23, Theorems 2.6 and 2.9].

5.1. An a priori estimate. In this subsection, we will establish an a priori estimate for all positive solutions of problem (1.3) which will play an important role in the proof of Theorem 1.3. 
First we introduce another ordered Banach subspace of $C(\bar{D})$ for the fixed point equation (3.7) which combines the good properties of the resolvent $K$ of problem (3.1) with the good properties of natural ordering of $C(\bar{D})$.

Let $\phi(x)=K 1(x)$ be the unique solution of problem (1.11). Then it follows from [18, Lemma 2.7] that the function $\phi(x)$ belongs to $C^{\infty}(\bar{D})$ and satisfies the conditions

$$
\begin{gathered}
\phi(x) \begin{cases}>0 & \text { if either } x \in D \text { or } x \in \partial D, a(x)>0, \\
=0 & \text { if } x \in \partial D, a(x)=0,\end{cases} \\
\frac{\partial \phi}{\partial v}(x)<0 \quad \text { if } x \in \partial D, a(x)=0 .
\end{gathered}
$$

By using the function $\phi(x)$, we can introduce a subspace of $C(\bar{D})$ as follows:

$$
C_{\phi}(\bar{D}):=\{u \in C(\bar{D}): \exists \text { a constant } c>0 \text { such that }-c \phi \preceq u \preceq c \phi\} .
$$

The space $C_{\phi}(\bar{D})$ is given a norm by the formula

$$
\|u\|_{\phi}=\inf \{c>0:-c \phi \preceq u \preceq c \phi\} .
$$

If we let

$$
P_{\phi}:=C_{\phi}(\bar{D}) \cap P=\left\{u \in C_{\phi}(\bar{D}): u \succeq 0\right\},
$$

then it is easy to verify that the space $C_{\phi}(\bar{D})$ is an ordered Banach space having the positive cone $P_{\phi}$ with nonempty interior. For $u, v \in C_{\phi}(\bar{D})$, the notation $u \gg v$ means that $u-v$ is an interior point of $P_{\phi}$. It follows from [18, Proposition 2.8] that $K$ maps $C_{\phi}(\bar{D})$ compactly into itself, and that $K$ is strongly positive, that is, $K g \gg 0$ for all $g \in P_{\phi} \backslash\{0\}$.

It is easy to see that a function $u(x)$ is a solution of problem (1.3) if and only if it satisfies the nonlinear operator equation

$$
u=\lambda K(f(u)) \text { in } C_{\phi}(\bar{D}) .
$$

However we know from [18, Theorem 0] that the first eigenvalue $\lambda_{1}$ of $\mathfrak{U}$ is positive and simple, with positive eigenfunction $\varphi_{1}(x)$ :

$$
A \varphi_{1}=\lambda_{1} \varphi_{1} \quad \text { in } D, \quad \varphi_{1}>0 \text { in } D, \quad B \varphi_{1}=0 \text { on } \partial D
$$

Without loss of generality, one may assume that

$$
\max _{\bar{D}} \varphi_{1}(x)=1
$$

We let

$$
\gamma=\min \left\{\frac{f\left(t_{1}(\varepsilon)\right)}{t_{1}(\varepsilon)}: 0<\varepsilon<\frac{1}{4}\right\} .
$$

Here we remark that $t_{1}(\varepsilon) \rightarrow 1$ as $\varepsilon \downarrow 0$, so that the constant $\gamma$ is positive. 


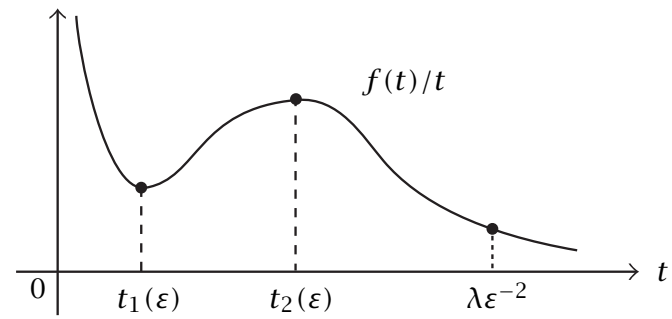

FIGURE 5.1

Then we have the following a priori estimate for all positive solutions $u$ of problem (1.3).

Proposition 5.1. There exists a constant $0<\varepsilon_{0} \leq 1 / 4$ such that if $\lambda>\lambda_{1} / \gamma$ and $0<\varepsilon \leq \varepsilon_{0}$, then for all positive solutions $u$ of problem (1.3),

$$
u \succeq \lambda \varepsilon^{-2} \varphi_{1}
$$

Proof. (i) Let $c$ be a parameter satisfying $0<c<1$. Then

$$
A\left(\lambda c \varepsilon^{-2} \varphi_{1}\right)-\lambda f\left(\lambda c \varepsilon^{-2} \varphi_{1}\right)=\lambda c \varepsilon^{-2} \varphi_{1}\left(\lambda_{1}-\lambda \frac{f\left(\lambda c \varepsilon^{-2} \varphi_{1}\right)}{\lambda c \varepsilon^{-2} \varphi_{1}}\right) \text { in } D
$$

However, since we have (see Figure 5.1)

$$
\frac{f(t)}{t} \longrightarrow 0 \quad \text { as } t \rightarrow \infty, \quad \frac{f(t)}{t} \longrightarrow \infty \quad \text { as } t \rightarrow 0
$$

it follows that

$$
\frac{f\left(\lambda c \varepsilon^{-2} \varphi_{1}\right)}{\lambda c \varepsilon^{-2} \varphi_{1}} \geq \min \left\{\frac{f\left(t_{1}(\varepsilon)\right)}{t_{1}(\varepsilon)}, \frac{f\left(\lambda \varepsilon^{-2}\right)}{\lambda \varepsilon^{-2}}\right\} \text { in } D .
$$

First we obtain from formula (5.8) that

$$
\lambda_{1}-\lambda \frac{f\left(t_{1}(\varepsilon)\right)}{t_{1}(\varepsilon)} \leq \lambda_{1}-\lambda \gamma<0 \quad \forall \lambda>\frac{\lambda_{1}}{\gamma}, 0<\varepsilon<\frac{1}{4} .
$$

Secondly we have, for all $\lambda>\lambda_{1} / \gamma$,

$$
\lambda_{1}-\lambda \frac{f\left(\lambda \varepsilon^{-2}\right)}{\lambda \varepsilon^{-2}}=\lambda_{1}-\varepsilon^{2} \exp \left[\frac{1}{\varepsilon+\varepsilon^{2} / \lambda}\right] \leq \lambda_{1}-\varepsilon^{2} \exp \left[\frac{1}{\varepsilon+\varepsilon^{2} \gamma / \lambda_{1}}\right]
$$

However, one can find a constant $\varepsilon_{0} \in(0,1 / 4]$ such that, for all $0<\varepsilon \leq \varepsilon_{0}$,

$$
\lambda_{1}-\varepsilon^{2} \exp \left[\frac{1}{\varepsilon+\varepsilon^{2} \gamma / \lambda_{1}}\right]<0 .
$$


Hence it follows that

$$
\lambda_{1}-\lambda \frac{f\left(\lambda \varepsilon^{-2}\right)}{\lambda \varepsilon^{-2}}<0 \quad \forall \lambda>\frac{\lambda_{1}}{\gamma}, 0<\varepsilon \leq \varepsilon_{0} .
$$

Therefore, combining inequalities (5.12), (5.13), and (5.16) we obtain that, for all $\lambda>\lambda_{1} / \gamma$ and $0<\varepsilon \leq \varepsilon_{0}$,

$$
\begin{aligned}
A\left(\lambda c \varepsilon^{-2} \varphi_{1}\right)-\lambda f\left(\lambda c \varepsilon^{-2} \varphi_{1}\right) & =\lambda c \varepsilon^{-2} \varphi_{1}\left(\lambda_{1}-\lambda \frac{f\left(\lambda c \varepsilon^{-2} \varphi_{1}\right)}{\lambda c \varepsilon^{-2} \varphi_{1}}\right) \\
& \leq \lambda c \varepsilon^{-2} \varphi_{1}\left(\lambda_{1}-\lambda \min \left\{\frac{f\left(t_{1}(\varepsilon)\right)}{t_{1}(\varepsilon)}, \frac{f\left(\lambda \varepsilon^{-2}\right)}{\lambda \varepsilon^{-2}}\right\}\right) \\
& <0 \quad \text { in } D
\end{aligned}
$$

so that

$$
\lambda f\left(\lambda c \varepsilon^{-2} \varphi_{1}\right)>A\left(\lambda c \varepsilon^{-2} \varphi_{1}\right) \text { in } D .
$$

By applying the resolvent $K$ to the both sides, we have, for all $\lambda>\lambda_{1} / \gamma$ and $0<\varepsilon \leq \varepsilon_{0}$,

$$
\lambda K\left(f\left(\lambda c \varepsilon^{-2} \varphi_{1}\right)\right) \gg \lambda c \varepsilon^{-2} \varphi_{1} .
$$

(ii) Now we need the following lemma (cf. Wiebers [23, Lemma 1.3]).

LEMMA 5.2. If there exist a function $\tilde{u} \gg 0$ and a constant $s_{0}>0$ such that $\lambda K(f(s \tilde{u})) \gg s \tilde{u}$ for all $0 \leq s<s_{0}$, then for each fixed point $u$ of the mapping $\lambda K(f(u))$,

$$
u \succeq s_{0} \tilde{u} .
$$

Proof. Assume to the contrary that there exists a fixed point $u$ of $\lambda K(f(\cdot))$ with $u \nsucceq s_{0} \tilde{u}$. Then we can choose a constant $0 \leq \tilde{s}<s_{0}$ such that

$$
u-\tilde{s} \tilde{u} \in \partial P_{\phi} .
$$

However, since $\tilde{s} \tilde{u}$ satisfies the condition

$$
\lambda K(f(\tilde{s} \tilde{u})) \gg \tilde{s} \tilde{u}
$$

it follows from condition (5.21) that

$$
u=\lambda K(f(u)) \geq \lambda K(f(\tilde{s} \tilde{u})) \gg \tilde{s} \tilde{u},
$$

so that

$$
u-\tilde{s} \tilde{u} \in \stackrel{\circ}{P}_{\phi}
$$

This contradicts condition (5.21).

(iii) Since $\lambda K(f(0)) \gg 0$ and estimate (5.19) holds for all $0<c<1$, it follows from an application of Lemma 5.2 with $\tilde{u}:=\lambda \varepsilon^{-2} \varphi_{1}, s_{0}:=1$, and $s:=c$ (and also (5.5)) that 


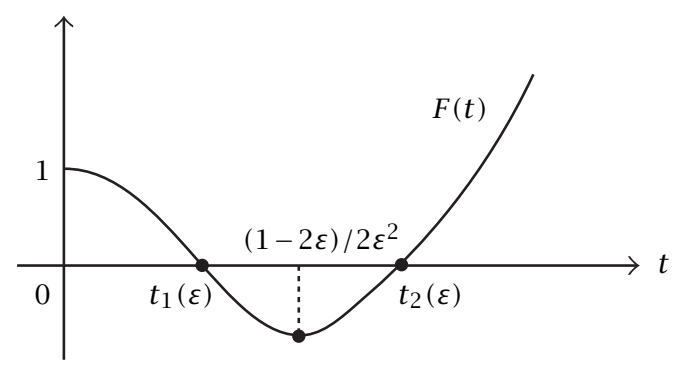

FIGURE 5.2

every positive solution $u$ of problem (1.3) satisfies the estimate

$$
u \succeq \lambda \varepsilon^{-2} \varphi_{1} \quad \forall \lambda>\frac{\lambda_{1}}{\gamma}, 0<\varepsilon \leq \varepsilon_{0} .
$$

The proof of Proposition 5.1 is complete.

5.2. End of proof of Theorem 1.3. (I) First we introduce a function

$$
F(t):=f(t)-f^{\prime}(t) t=\frac{\varepsilon^{2} t^{2}+(2 \varepsilon-1) t+1}{(1+\varepsilon t)^{2}} \exp \left[\frac{t}{1+\varepsilon t}\right], \quad t \geq 0 .
$$

The next lemma summarizes some elementary properties of the function $F(t)$.

LEMmA 5.3. Let $0<\varepsilon<1 / 4$. Then the function $F(t)$ has the properties

$$
F(t) \begin{cases}>0 & \text { if either } 0 \leq t<t_{1}(\varepsilon) \text { or } t>t_{2}(\varepsilon), \\ =0 & \text { if } t=t_{1}(\varepsilon), t=t_{2}(\varepsilon), \\ <0 & \text { if } t_{1}(\varepsilon)<t<t_{2}(\varepsilon) .\end{cases}
$$

Moreover, the function $F(t)$ is decreasing in the interval $\left(0,(1-2 \varepsilon) / 2 \varepsilon^{2}\right)$ and is increasing in the interval $\left((1-2 \varepsilon) / 2 \varepsilon^{2}, \infty\right)$, and has a minimum at $t=(1-2 \varepsilon) / 2 \varepsilon^{2}$ (see Figure 5.2).

(II) The next proposition is an essential step in the proof of Theorem 1.3 (cf. Amann [1, Lemma 7.8]).

Proposition 5.4. Let $0<\varepsilon<1 / 4$. Then there exists a constant $\alpha>0$, independent of $\varepsilon$, such that for all $u \succeq \alpha \varepsilon^{-2} \varphi_{1}$,

$$
K(F(u)) \gg 0 .
$$

Proof. First, since $t_{2}(\varepsilon)<2 \varepsilon^{-2}$, it follows from Lemma 5.3 that

$$
F(t) \geq F\left(2 \varepsilon^{-2}\right)>0 \quad \forall t \geq 2 \varepsilon^{-2} .
$$


We define two functions

$$
\begin{aligned}
& z_{-}(u)(x)= \begin{cases}-F(u(x)) & \text { if } u(x) \geq 2 \varepsilon^{-2}, \\
0 & \text { if } u(x)<2 \varepsilon^{-2},\end{cases} \\
& z_{+}(u)(x)=F(u(x))+z_{-}(u)(x) .
\end{aligned}
$$

Moreover, we define two sets

$$
M:=\left\{x \in \bar{D}: \varphi_{1}(x)>\frac{1}{2}\right\}, \quad L:=\left\{x \in \bar{D}: u(x) \geq 2 \varepsilon^{-2}\right\}
$$

Then $M \subset L$ for all $u \succeq 4 \varepsilon^{-2} \varphi_{1}$, and so

$$
z_{-}(u) \leq-F\left(2 \varepsilon^{-2}\right) \chi_{L} \leq-F\left(2 \varepsilon^{-2}\right) \chi_{M} .
$$

By using Friedrichs' mollifiers, we can construct a function $v(x) \in C^{\infty}(\bar{D})$ such that $v \succ 0$ and that

$$
z_{-}(u) \leq-F\left(2 \varepsilon^{-2}\right) v \quad \forall u \succeq 4 \varepsilon^{-2} \varphi_{1}
$$

On the other hand, by Lemma 5.3 we remark that

$$
\min \left\{F(t): 0 \leq t \leq 2 \varepsilon^{-2}\right\}=F\left(\frac{1-2 \varepsilon}{2 \varepsilon^{2}}\right)<0 .
$$

Since we have

$$
z_{+}(u)(x)= \begin{cases}0 & \text { if } x \in L, \\ F(u(x)) & \text { if } x \notin L,\end{cases}
$$

it follows that

$$
z_{+}(u) \geq F\left(\frac{1-2 \varepsilon}{2 \varepsilon^{2}}\right) \chi_{\bar{D} \backslash L} .
$$

If $\alpha$ is a constant greater than 4 , we define a set

$$
M_{\alpha}:=\left\{x \in \bar{D}: \varphi_{1}(x)<\frac{2}{\alpha}\right\}
$$

Then we have, for all $u \succeq \alpha \varepsilon^{-2} \varphi_{1}$,

$$
\bar{D} \backslash L=\left\{x \in \bar{D}: u(x)<2 \varepsilon^{-2}\right\} \subset M_{\alpha},
$$

and hence

$$
z_{+}(u) \geq F\left(\frac{1-2 \varepsilon}{2 \varepsilon^{2}}\right) \chi_{M_{\alpha}} \quad \forall u \succeq \alpha \varepsilon^{-2} \varphi_{1} .
$$


Thus, combining inequalities (5.33) and (5.39) we obtain that

$$
\begin{aligned}
K(F(u)) & =K\left(z_{+}(u)-z_{-}(u)\right) \\
& \geq F\left(\frac{1-2 \varepsilon}{2 \varepsilon^{2}}\right) K\left(\chi_{M_{\alpha}}\right)+F\left(2 \varepsilon^{-2}\right) K v \quad \forall u \succeq \alpha \varepsilon^{-2} \varphi_{1}
\end{aligned}
$$

However, by [18, estimate (2.11)] it follows that there exists a constant $c_{0}>0$ such that

$$
K v \succeq \mathcal{c}_{0} \varphi_{1}
$$

Furthermore, since $\chi_{M_{\alpha}} \rightarrow 0$ in $L^{p}(D)$ as $\alpha \rightarrow \infty$, it follows that $K\left(\chi_{M_{\alpha}}\right) \rightarrow 0$ in $C^{1}(\bar{D})$ and so $K\left(\chi_{M_{\alpha}}\right) \rightarrow 0$ in $C_{\phi}(\bar{D})$. Hence, for any positive integer $k$ one can choose the constant $\alpha$ so large that

$$
K\left(\chi_{M_{\alpha}}\right) \preceq \frac{c_{0}}{k} \varphi_{1} .
$$

Thus, carrying inequalities (5.41) and (5.42) into the right-hand side of inequality (5.40) we obtain that

$$
\begin{aligned}
K(F(u)) & =K\left(z_{+}(u)-z_{-}(u)\right) \\
& \geq F\left(\frac{1-2 \varepsilon}{2 \varepsilon^{2}}\right) \frac{c_{0}}{k} \varphi_{1}+F\left(2 \varepsilon^{-2}\right) \mathcal{c}_{0} \varphi_{1} \\
& =F\left(2 \varepsilon^{-2}\right) \mathcal{c}_{0} \varphi_{1}\left(1+\frac{F\left((1-2 \varepsilon) / 2 \varepsilon^{2}\right)}{F\left(2 \varepsilon^{-2}\right)} \frac{1}{k}\right) \quad \forall u \succeq \alpha \varepsilon^{-2} \varphi_{1} .
\end{aligned}
$$

However we have, as $\varepsilon \downarrow 0$,

$$
\frac{F\left((1-2 \varepsilon) / 2 \varepsilon^{2}\right)}{F\left(2 \varepsilon^{-2}\right)}=\frac{(4 \varepsilon-1)(\varepsilon+2)^{2}}{\varepsilon^{2}+4 \varepsilon+2} \exp \left[\frac{-2 \varepsilon-3}{\varepsilon+2}\right] \rightarrow-2 e^{-3 / 2}
$$

Therefore the desired inequality (5.28) follows from inequality (5.43) if we take the positive integer $k$ so large that

$$
k>-\min _{0<\varepsilon<1 / 4} \frac{F\left((1-2 \varepsilon) / 2 \varepsilon^{2}\right)}{F\left(2 \varepsilon^{-2}\right)} .
$$

The proof of Proposition 5.4 is complete.

(III) Proposition 5.4 implies the following important property of the nonlinear mapping $K(f(\cdot))$ (cf. Wiebers [23, Lemma 2.2]).

LEMMA 5.5. Let $0<\varepsilon<1 / 4$ and let $\alpha$ be the same constant as in Proposition 5.4. Then for all $u \succeq \alpha \varepsilon^{-2} \varphi_{1}$ and all $s>1$,

$$
s K(f(u)) \gg K(f(s u)) .
$$


Proof. By Taylor's formula, it follows that

$$
\begin{aligned}
& s K(f(u))-K(f(s u)) \\
& \quad=s K(f(u))-K(f(u))+K\left(f^{\prime}(u)(s u-u)+o(\|s u-u\|)\right) \\
& \quad=(s-1)\left(K(F(u))-\frac{o(\|s u-u\|)}{s-1}\right) .
\end{aligned}
$$

However Proposition 5.4 tells us that there exists an element $\hat{v} \in \stackrel{\circ}{P}_{\phi}$ such that

$$
K(F(u)) \succeq \hat{v} \quad \forall u \succeq \alpha \varepsilon^{-2} \varphi_{1} .
$$

Now let $\mathscr{A}$ be an arbitrary compact subset of $\alpha \varepsilon^{-2} \varphi_{1}+P_{\phi}$. Then, by combining inequalities (5.47) and (5.48) one can find a constant $s_{0}>1$ such that

$$
s K(f(u))-K(f(s u)) \gg(s-1)\left(\hat{v}-\frac{o(\|s u-u\|)}{s-1}\right) \quad \forall u \in \mathscr{A}, \forall 1<s \leq s_{0} .
$$

In particular, if $s>1$ and $u \succeq \alpha \varepsilon^{-2} \varphi_{1}$, we let

$$
\mathscr{A}:=\{\sigma u: 1 \leq \sigma \leq s\}, \quad s:=t .
$$

By inequality (5.49), we have, for all $1<t \leq s_{0}$ and all $1 \leq \sigma \leq s$,

$$
t K(f(\sigma u)) \gg K(f(t \sigma u)) .
$$

It should be noticed that, for given $s>1$, there exist numbers $1<t_{1} \leq t_{2} \leq \cdots \leq t_{m} \leq$ $s_{0}$ with

$$
\prod_{i=1}^{m} t_{i}=s .
$$

Therefore, by using inequality (5.51) $m$-times we obtain that

$$
\begin{aligned}
K(f(s u)) & =K\left(f\left(\prod_{i=1}^{m} t_{i} u\right)\right) \\
& \ll t_{1} K\left(f\left(\prod_{i=2}^{m} t_{i} u\right)\right) \cdots \ll \prod_{i=1}^{m} t_{i} K(f(u)) \\
& =s K(f(u)) .
\end{aligned}
$$

This proves Lemma 5.5 .

(IV) If $\varepsilon_{0}$ and $\alpha$ are the constants as in Propositions 5.1 and 5.4, respectively, then we let

$$
\Lambda_{1}:=\max \left\{\frac{\lambda_{1}}{\gamma}, \alpha\right\}
$$

If $u_{1}=u_{1}(\lambda)$ and $u_{2}=u_{2}(\lambda)$ are two positive solutions of problem (1.3) with $\lambda>\Lambda_{1}$ and $0<\varepsilon \leq \varepsilon_{0}$, then combining Proposition 5.1 and Lemma 5.5 we find that, for all $s>1$,

$$
s K\left(f\left(u_{i}\right)\right) \gg K\left(f\left(s u_{i}\right)\right), \quad i=1,2,
$$


so that

$$
s u_{i}=s \lambda K\left(f\left(u_{i}\right)\right) \gg \lambda K\left(f\left(s u_{i}\right)\right), \quad i=1,2 .
$$

Therefore we obtain that $u_{1}=u_{2}$, by applying the following lemma with $\tilde{u}:=u_{1}$ and $u:=u_{2}$ and with $\tilde{u}:=u_{2}$ and $u:=u_{1}$ (see Wiebers [23, Lemma 1.3]).

LEMMA 5.6. If there exists a function $\tilde{u} \gg 0$ such that $s \tilde{u} \gg \lambda K(f(s \tilde{u}))$ for all $s>1$, then $\tilde{u} \succeq u$ for each fixed point $u$ of the mapping $\lambda K(f(\cdot))$.

Proof. Assume to the contrary that there exists a fixed point $u$ of $\lambda K(f(\cdot))$ with $\tilde{u} \nsucceq u$. Then we can choose a constant $\tilde{s}>1$ such that

$$
\tilde{s} \tilde{u}-u \in \partial P_{\phi} .
$$

However, since $\tilde{s} \tilde{u}$ satisfies the condition

$$
\tilde{s} \tilde{u} \gg \lambda K(f(\tilde{s} \tilde{u})),
$$

it follows from condition (5.57) that

$$
\tilde{s} \tilde{u} \gg \lambda K(f(\tilde{s} \tilde{u})) \succeq \lambda K(f(u))=u,
$$

so that

$$
\tilde{s} \tilde{u}-u \in \stackrel{\circ}{P}_{\phi} .
$$

This contradicts condition (5.57).

(V) Finally it remains to consider the case where $\varepsilon_{0}<\varepsilon<1 / 4$. If $u(\lambda)$ is a positive solution of problem (1.3), then

$$
A\left(u(\lambda)-\frac{\lambda}{\lambda_{1}} \varphi_{1}\right)=\lambda f(u(\lambda))-\lambda \varphi_{1} \geq \lambda\left(1-\varphi_{1}\right) \geq 0 \quad \text { in } D .
$$

By the positivity of the resolvent $K$, it follows that

$$
u(\lambda) \geq \frac{\lambda}{\lambda_{1}} \varphi_{1} \geq \frac{\alpha}{\varepsilon^{2}} \varphi_{1} \quad \forall \lambda \geq \frac{\alpha \lambda_{1}}{\varepsilon^{2}} .
$$

Therefore, just as in the case $0<\varepsilon \leq \varepsilon_{0}$, we can prove that the uniqueness result for positive solutions of problem (1.3) holds true if we take the parameter $\lambda$ so large that

$$
\lambda \geq \Lambda_{2}:=\frac{\alpha \lambda_{1}}{\varepsilon^{2}} .
$$

Now the proof of Theorem 1.3 is complete if we take $\Lambda=\max \left\{\Lambda_{1}, \Lambda_{2}\right\}$.

6. Proof of Theorem 1.4. Our proof is based on a method inspired by Dancer [10, Theorem 1].

(I) First we prove assertion (1.19). Let $u(\lambda)$ be the unique positive solution of problem (1.3) for $\lambda$ sufficiently small as in Theorem 1.2

$$
0<\lambda<\frac{\lambda_{1} \exp [(2 \varepsilon-1) / \varepsilon]}{4 \varepsilon^{2}} .
$$


Let $\phi(x)=K 1(x)$ be the unique positive solution of the linear eigenvalue problem (1.11). Then it is easy to see that, for all $\lambda>0$, the functions $\lambda \phi(x)$ and $\lambda e^{1 / \varepsilon} \phi(x)$ are a subsolution and a supersolution of problem (1.3), respectively. Indeed, since the function

$$
f(t)=\exp \left[\frac{t}{1+\varepsilon t}\right]
$$

is increasing for all $t \geq 0$, and satisfies the condition

$$
f(0)=1<f(t)<f(\infty)=e^{1 / \varepsilon}, \quad t>0 .
$$

it follows that

$$
A(\lambda \phi)=\lambda<\lambda f(\lambda \phi) \quad \text { in } D, \quad B(\lambda \phi)=0 \quad \text { on } \partial D
$$

and that

$$
A\left(\lambda e^{1 / \varepsilon} \phi\right)=\lambda e^{1 / \varepsilon}>\lambda f\left(\lambda e^{1 / \varepsilon} \phi\right) \quad \text { in } D, \quad B\left(\lambda e^{1 / \varepsilon} \phi\right)=0 \quad \text { on } \partial D .
$$

Hence, by applying the method of super-subsolutions (see [18, Theorem 2]) to our situation one can find a solution $v(\lambda) \in C^{2}(\bar{D})$ of problem (1.3) such that

$$
\lambda \phi(x) \leq v(\lambda)(x) \leq \lambda e^{1 / \varepsilon} \phi(x) \quad \text { on } \bar{D} .
$$

However, Theorems 1.2 and 1.3 tell us that problem (1.3) has a unique positive solution $u(\lambda) \in C^{2}(\bar{D})$ if $\lambda$ is either sufficiently small or sufficiently large. Therefore it follows from assertion (6.6) that $v(\lambda)=u(\lambda)$ in $D$, and so we have, for $\lambda$ sufficiently small and sufficiently large,

$$
\lambda \phi(x) \leq u(\lambda)(x) \leq \lambda e^{1 / \varepsilon} \phi(x) \text { on } \bar{D} .
$$

By assertion (6.7), we have, for all $x \in \bar{D}$,

$$
u(\lambda)(x) \longrightarrow 0 \text { as } \lambda \downarrow 0 .
$$

Hence, applying the Lebesgue convergence theorem we obtain from condition (6.3) that

$$
f(u(\lambda)) \longrightarrow f(0)=1 \quad \text { in } L^{p}(D) \text { as } \lambda \downarrow 0 .
$$

On the other hand, it follows from Theorem 3.1 that the resolvent $K$ maps $L^{p}(D)$ continuously into $W^{2, p}(D)$.

Hence we have, by assertion (6.9),

$$
\frac{u(\lambda)}{\lambda}=K f(u(\lambda)) \longrightarrow K 1=\phi \quad \text { in } W^{2, p}(D) \text { as } \lambda \downarrow 0 .
$$

By Sobolev's imbedding theorem, this proves that

$$
\frac{u(\lambda)}{\lambda} \rightarrow \phi \quad \text { in } C^{1}(\bar{D}) \text { as } \lambda \downarrow 0
$$

Indeed, it suffices to note that $W^{2, p}(D) \subset C^{1}(\bar{D})$ if we take $p>N$ and so $2-N / p>1$. 
(II) Secondly we prove assertion (1.20). The proof is carried out in the same way as in the proof of assertion (1.19).

Let $u(\lambda)$ be the unique positive solution for $\lambda$ sufficiently large as in Theorem 1.3

$$
\lambda>\Lambda, \quad \Lambda \sim \frac{v\left(t_{1}(\varepsilon)\right)}{\|\phi\|_{\infty}} .
$$

Since we have, for all $x \in D$,

$$
\phi(x)>0,
$$

it follows from assertion (6.7) that, for all $x \in D$,

$$
u(\lambda)(x) \rightarrow \infty \text { as } \lambda \uparrow \infty .
$$

Therefore, just as in step (I) we obtain assertion (6.3) that

$$
\frac{u(\lambda)}{\lambda}=K f(u(\lambda)) \rightarrow K f(\infty)=e^{1 / \varepsilon} \phi \quad \text { in } C^{1}(\bar{D}) \text { as } \lambda \uparrow \infty .
$$

The proof of Theorem 1.4 is complete.

ACKNOwledgement. The author is grateful to Kenichiro Umezu-a former student, now collaborator-for fruitful conversations while working on this paper.

\section{REFERENCES}

[1] H. Amann, Multiple positive fixed points of asymptotically linear maps, J. Functional Analysis 17 (1974), 174-213. MR 50\#3020. Zbl 0287.47037.

[2] __ Fixed point equations and nonlinear eigenvalue problems in ordered Banach spaces, SIAM Rev. 18 (1976), no. 4, 620-709. MR 54\#3519. Zbl 0345.47044.

[3] R. Aris, The Mathematical Theory of Diffusion and Reaction in Permeable Catalysts. I. The Theory of the Steady State, Clarendon Press, Oxford, 1975. Zbl 0315.76051.

[4] _ The Mathematical Theory of Diffusion and Reaction in Permeable Catalysts. II. Questions of Uniqueness, Stability, and Transient Behaviour, Clarendon Press, Oxford, 1975. Zbl 0315.76052.

[5] J. Bebernes and D. Eberly, Mathematical Problems from Combustion Theory, Applied Mathematical Sciences, vol. 83, Springer-Verlag, New York, 1989. MR 91d:35165. Zbl 0692.35001.

[6] T. Boddington, P. Gray, and G. C. Wake, Criteria for thermal explosions with and without reactant consumption, Proc. Roy. Soc. London Ser. A 357 (1977), 403-422.

[7] K. J. Brown, M. M. A. Ibrahim, and R. Shivaji, S-shaped bifurcation curves, Nonlinear Anal. 5 (1981), no. 5, 475-486. MR 82h:35007. Zbl 0458.35036.

[8] D. S. Cohen, Multiple stable solutions of nonlinear boundary value problems arising in chemical reactor theory, SIAM J. Appl. Math. 20 (1971), 1-13. MR $43 \# 606$. Zbl 0219.34027.

[9] D. S. Cohen and T. W. Laetsch, Nonlinear boundary value problems suggested by chemical reactor theory, J. Differential Equations 7 (1970), 217-226. MR 41\#3994. Zbl 0201.43102.

[10] E. N. Dancer, On the number of positive solutions of weakly nonlinear elliptic equations when a parameter is large, Proc. London Math. Soc. (3) 53 (1986), no. 3, 429-452. MR 88c:35061. Zbl 0572.35040.

[11] J. Dugundji, An extension of Tietze's theorem, Pacific J. Math. 1 (1951), 353-367. MR 13,373c. Zbl 0043.38105.

[12] _ Topology, Allyn and Bacon, Massachusetts, 1966. MR 33\#1824. Zbl 0144.21501. 
[13] R. W. Leggett and L. R. Williams, Multiple positive fixed points of nonlinear operators on ordered Banach spaces, Indiana Univ. Math. J. 28 (1979), no. 4, 673-688. MR 80i:47073. Zbl 0421.47033.

[14] T. Minamoto, N. Yamamoto, and M. T. Nakao, Numerical verification method for solutions of the perturbed Gelfand equation, Methods Appl. Anal. 7 (2000), no. 1, 251-262. MR 2001i:34031.

[15] S. V. Parter, Solutions of a differential equation arising in chemical reactor processes, SIAM J. Appl. Math. 26 (1974), 687-716. MR 52\#3654. Zbl 0285.34013.

[16] M. H. Protter and H. F. Weinberger, Maximum Principles in Differential Equations, PrenticeHall, New Jersey, 1967. MR 36\#2935.

[17] K. Taira, Analytic Semigroups and Semilinear Initial-Boundary Value Problems, London Mathematical Society Lecture Note Series, vol. 223, Cambridge University Press, Cambridge, 1995. MR 97g:47035. Zbl 0861.35001.

[18] _ Bifurcation theory for semilinear elliptic boundary value problems, Hiroshima Math. J. 28 (1998), no. 2, 261-308. MR 99j:35071. Zbl 0937.35059.

[19] K. Taira and K. Umezu, Positive solutions of sublinear elliptic boundary value problems, Nonlinear Anal. 29 (1997), no. 7, 761-771. MR 98h:35082. Zbl 0878.35048.

[20] _ Semilinear elliptic boundary value problems in chemical reactor theory, J. Differential Equations 142 (1998), no. 2, 434-454. MR 99c:35080. Zbl 0917.35040.

[21] K. K. Tam, Construction of upper and lower solutions for a problem in combustion theory, J. Math. Anal. Appl. 69 (1979), no. 1, 131-145. MR 80h:80005. Zbl 0414.35011.

[22] J. Warnatz, U. Maas, and R. W. Dibble, Combustion, 2nd ed., Springer-Verlag, Berlin, 1999.

[23] H. Wiebers, S-shaped bifurcation curves of nonlinear elliptic boundary value problems, Math. Ann. 270 (1985), no. 4, 555-570. MR 86f:35027. Zbl 0544.35015.

[24]__ Critical behaviour of nonlinear elliptic boundary value problems suggested by exothermic reactions, Proc. Roy. Soc. Edinburgh Sect. A 102 (1986), no. 1-2, 19-36. MR 87k:35095. Zbl 0609.35073.

[25] L. R. Williams and R. W. Leggett, Multiple fixed point theorems for problems in chemical reactor theory, J. Math. Anal. Appl. 69 (1979), no. 1, 180-193. MR 80i:47083. Zbl 0416.47026.

KAZUAKI TAIRA: INSTITUTE OF MATHEMATICS, UNIVERSITY OF TSUKUBA, TSUKUBA 305-8571, JAPAN

E-mail address: taira@math.tsukuba.ac.jp 


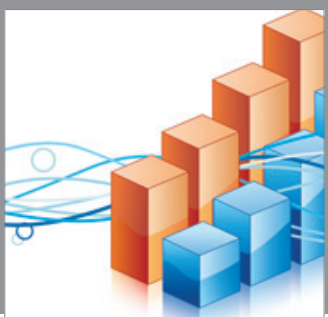

Advances in

Operations Research

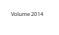

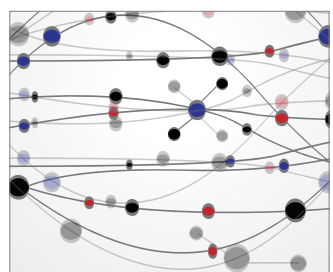

\section{The Scientific} World Journal
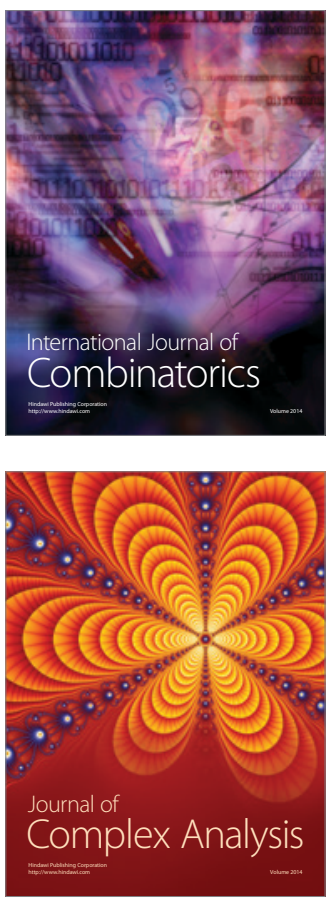

International Journal of

Mathematics and

Mathematical

Sciences
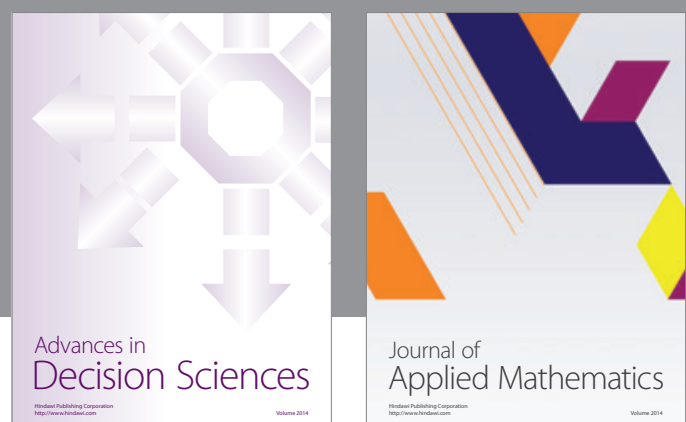

Journal of

Applied Mathematics
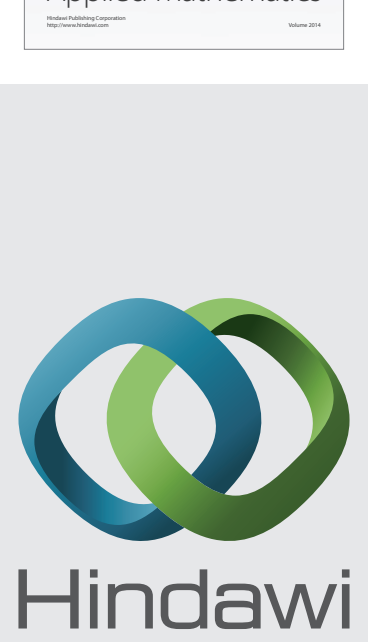

Submit your manuscripts at http://www.hindawi.com
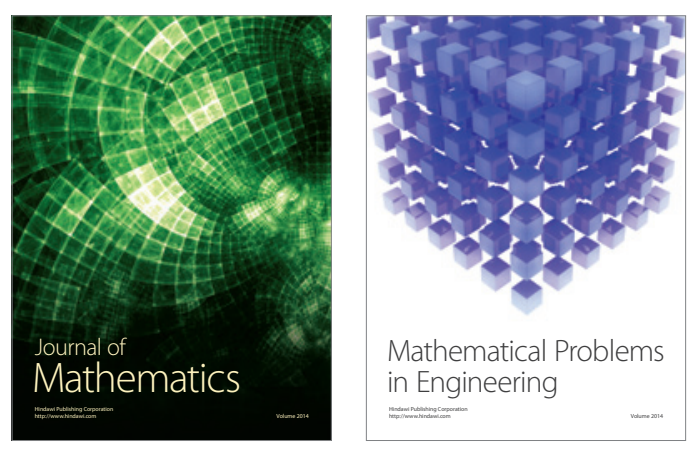

Mathematical Problems in Engineering
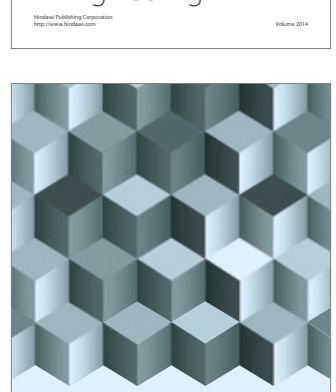

Journal of

Function Spaces
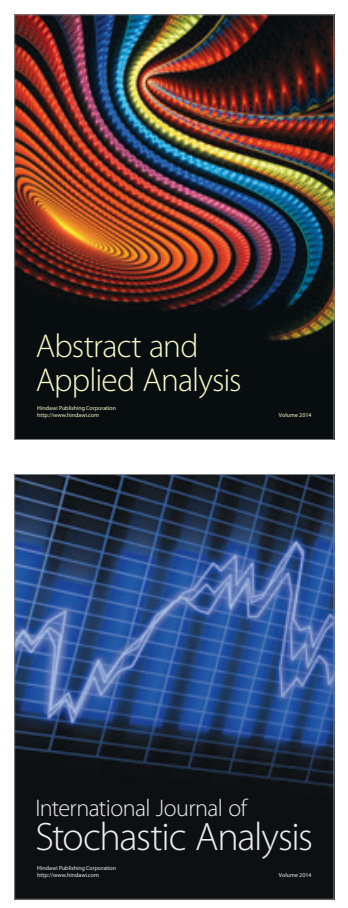

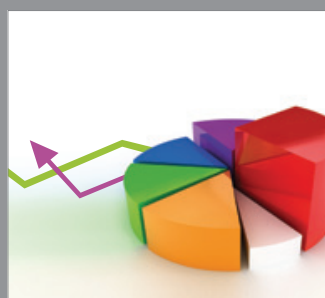

ournal of

Probability and Statistics

Promensencen
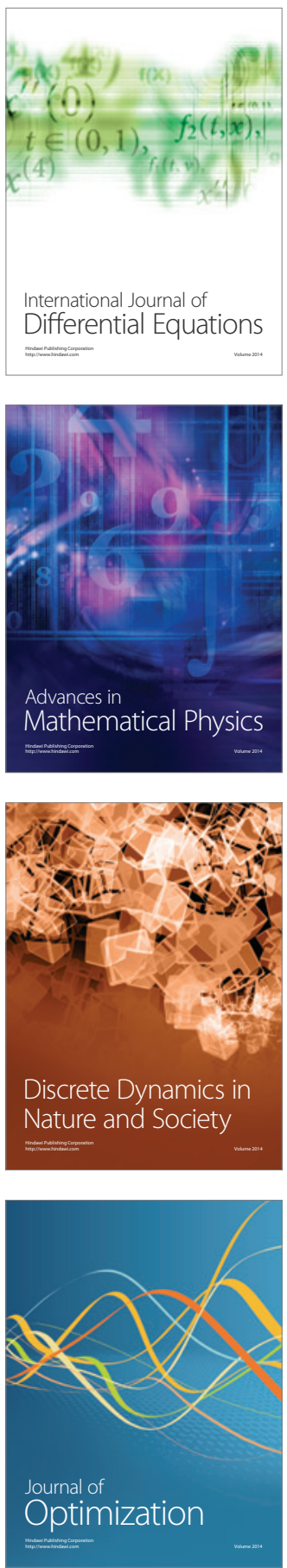